- Most mouth ulcers are inconsequential.

- Cancer and some systemic diseases may present with ulceration.

- Any single ulcer lasting three or more weeks should be regarded with suspicion.

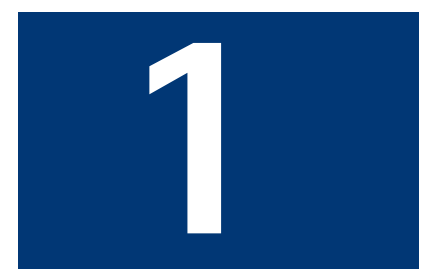

\title{
Oral medicine - Update for the dental practitioner Aphthous and other common ulcers
}

\author{
C. Scully ${ }^{1}$ and D. H. Felix ${ }^{2}$
}

This series provides an overview of current thinking in the more relevant areas of oral medicine for primary care practitioners, written by the authors while they were holding the Presidencies of the European Association for Oral Medicine and the British Society for Oral Medicine, respectively. A book containing additional material will be published. The series gives the detail necessary to assist the primary dental clinical team caring for patients with oral complaints that may be seen in general dental practice. Space precludes inclusion of illustrations of uncommon or rare disorders, or discussion of disorders affecting the hard tissues. Approaching the subject mainly by the symptomatic approach - as it largely relates to the presenting complaint - was considered to be a more helpful approach for GDPs rather than taking a diagnostic category approach. The clinical aspects of the relevant disorders are discussed, including a brief overview of the aetiology, detail on the clinical features and how the diagnosis is made. Guidance on management and when to refer is also provided, along with relevant websites which offer further detail.

\section{ORAL MEDICINE \\ 1. Aphthous and other common ulcers \\ 2. Mouth ulcers of more serious connotation \\ 3. Dry mouth and disorders of salivation \\ 4. Oral malodour \\ 5. Oral white patches \\ 6. Oral red and hyperpigmented patches \\ 7. Orofacial sensation and movement \\ 8. Orofacial swellings and lumps \\ 9. Oral cancer \\ 10. Orofacial pain \\ *Professor, Consultant, Dean, Eastman Dental Institute for Oral Health Care Sciences, 256 Gray's Inn Road, UCL, University of London, London WC1X 8LD; ${ }^{2}$ Consultant, Senior Lecturer, Glasgow Dental Hospital and School, 378 Sauchiehall Street, Glasgow G2 3JZ Associate Dean for Postgraduate Dental Education, NHS Education for Scotland, 2nd Floor, Hanover Buildings, 66 Rose Street, Edinburgh EH2 2NN \\ ${ }^{*}$ Correspondence to: Professor Crispian Scully CBE \\ Email:c.scully@eastman.ucl.ac.uk}

\section{Refereed Paper}

๑ British Dental Journal 2005; 199 259-264
Specialist referral may be indicated if the practitioner feels:

- the diagnosis is unclear

- a serious diagnosis is possible

- systemic disease may be present

- unclear as to investigations indicated

- complex investigations unavailable in primary care are indicated

- unclear as to treatment indicated

- treatment is complex

- treatment requires agents not readily available

- unclear as to the prognosis

- the patient wishes this.

\section{ULCERATION}

Ulceration is a breach in the oral epithelium, which typically exposes nerve endings in the underlying lamina propria, resulting in pain or soreness, especially when eating spicy foods or

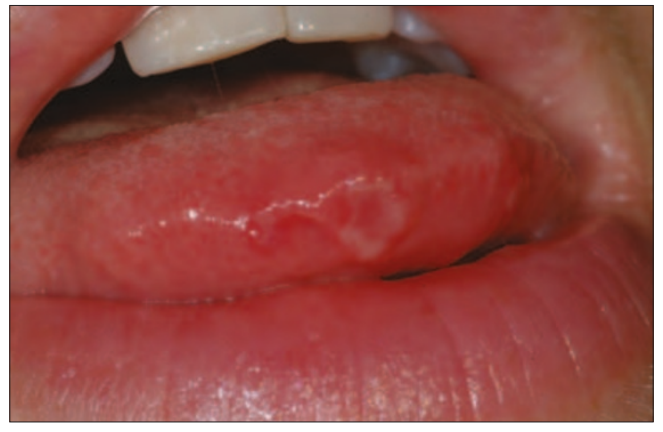

Fig. 1 A small erosion citrus fruits. Patients vary enormously in the degree to which they suffer and complain of soreness in relation to oral ulceration. It is always important to exclude serious disorders such as oral cancer (Part 9) or other serious disease, but not all patients who complain of soreness have discernible organic disease. Conversely, some with serious disease have no pain. Even in those with detectable lesions, the level of complaint can vary enormously. Some patients with large ulcers complain little; others with minimal ulceration complain bitterly of discomfort. Sometimes there is a psychogenic influence.

\section{Terminology}

Epithelial thinning or breaches may be seen in:

- mucosal atrophy or desquamation - terms often used for thinning of the epithelium which assumes a red appearance as the underlying lamina propria containing blood vessels shows

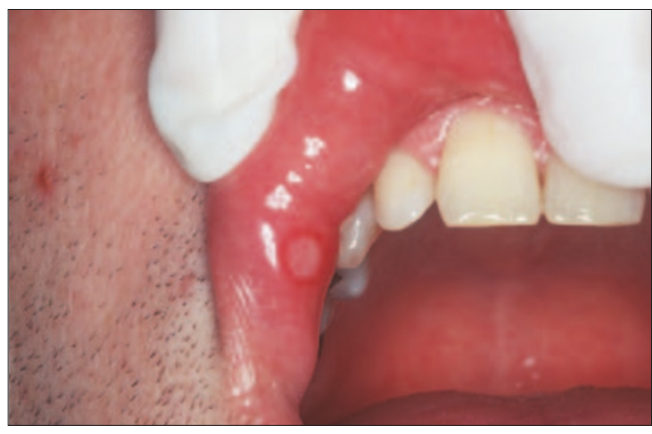

Fig. 2 Minor aphthous ulcer, labial mucosa 
through. Most commonly this is seen in desquamative gingivitis (usually related to lichen planus, or less commonly to pemphigoid) and in geographic tongue (erythema migrans, benign migratory glossitis). A similar process may also be seen in systemic disorders such as deficiency states (of iron, folic acid or B vitamins).

- mucosal inflammation (mucositis, stomatitis) which can cause soreness. Viral stomatitis, candidosis, radiation mucositis, chemotherapyrelated mucositis and graft-versus-host-disease are examples.

- erosion which is the term used for superficial breaches of the epithelium. These often have a red appearance initially as there is little damage to the underlying lamina propria, but they typically become covered by a fibrinous exudate which has a yellowish appearance (Fig. 1). Erosions are common in vesiculobullous disorders such as pemphigoid.

- ulcer which is the term usually used where there is damage both to epithelium and lamina propria. An inflammatory halo, if present, also highlights the ulcer with a red halo around the yellow or grey ulcer (Fig. 2). Most ulcers are due to local causes such as trauma or burns, but recurrent aphthous stomatitis and cancer must always be considered.
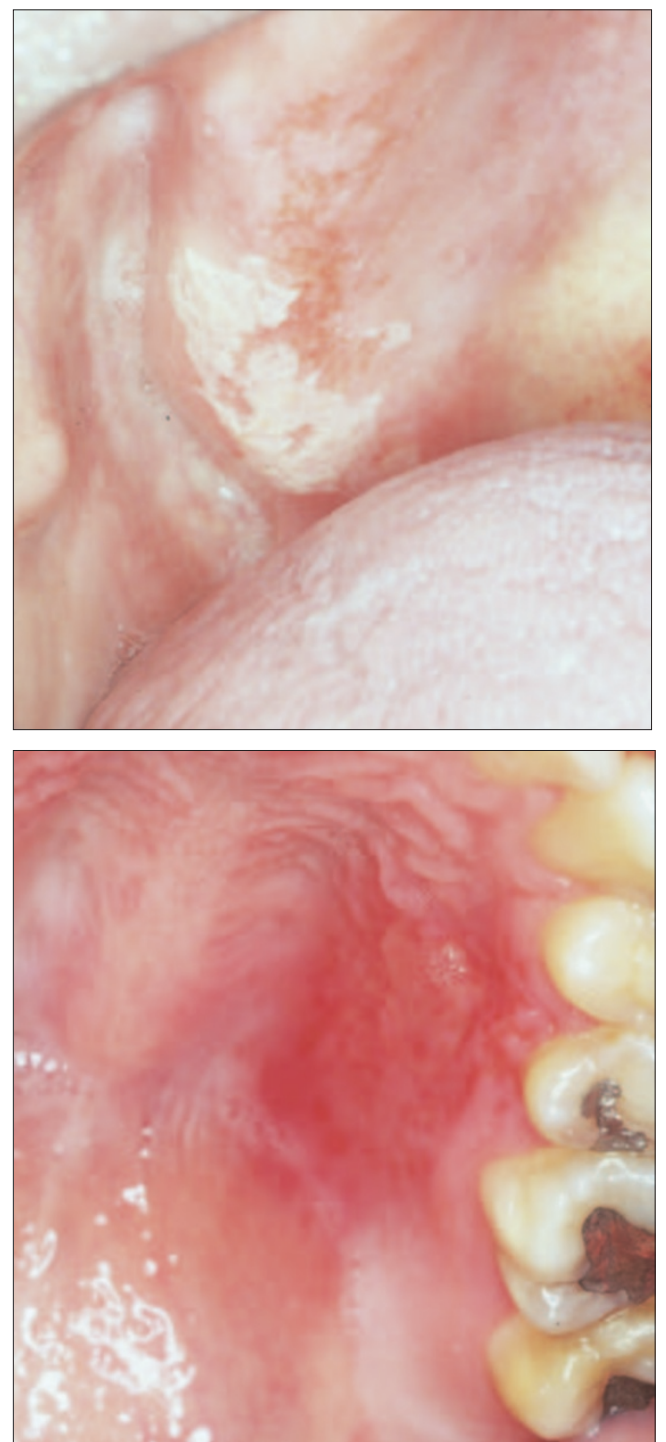

\section{Table 1 Main causes of oral ulceration}

Local causes

Aphthae

Infections

Drugs

Malignant disease

Systemic diseases

\section{Table 2 Main causes of mouth ulcers}

\section{Local causes}

Trauma

Appliances

latrogenic

Non-accidental injury

Self-inflicted

Sharp teeth or restorations

Burns

$$
\begin{aligned}
& \text { Chemical } \\
& \text { Cold } \\
& \text { Electric } \\
& \text { Heat }
\end{aligned}
$$

Radiation

\section{Recurrent aphthae}

Infections

Acute necrotising gingivitis

Chickenpox

Deep mycoses

Hand, foot and mouth disease

Herpangina

Herpetic stomatitis

HIV

Infectious mononucleosis

Syphilis

Tuberculosis

Drugs

Cytotoxic drugs,

Nicorandil, NSAIDs

Many others

Malignant neoplasms

Oral

Encroaching from antrum

\section{Systemic disease}

Mucocutaneous disease

Behcet's syndrome

Chronic ulcerative stomatitis

Epidermolysis bullosa

Erythema multiforme

Lichen planus

Pemphigus vulgaris

Sub-epithelial immune blistering diseases

(Pemphigoid and variants, dermatitis

herpetiformis, linear IgA disease)

Haematological disorders

Anaemia

Gammopathies

Haematinic deficiencies

Leukaemia and myelodysplastic syndrome

Neutropenia and other white cell dyscrasias

Gastrointestinal disease

Coeliac disease

Crohn's disease

Ulcerative colitis

\section{Miscellaneous uncommon diseases}

Eosinophilic ulcer

Giant cell arteritis

Hypereosinophilic syndrome

Lupus erythematosus

Necrotising sialometaplasia

Periarteritis nodosa

Reiters syndrome

Sweet's syndrome

Wegener's granulomatosis 


\section{Causes of oral ulceration}

Ulcers and erosions can also be the final common manifestation of a spectrum of conditions. These range from: epithelial damage resulting from trauma; an immunological attack as in lichen planus, pemphigoid or pemphigus; damage because of an immune defect as in HIV disease and leukaemia; infections such as herpesviruses, tuberculosis and syphilis; cancer and nutritional defects such as vitamin deficiencies and some gastrointestinal diseases (Tables 1 and 2).

\section{Ulcers of local causes}

At any age, there may be burns from chemicals of various kinds (Fig. 3), heat (Fig. 4), cold, or ionising radiation or factitious ulceration, especially of the maxillary gingivae or palate.

Children may develop ulceration of the lower lip by accidental biting following dental local anaesthesia. Ulceration of the upper labial fraenum, especially in a child with bruised and swollen lips, subluxed teeth or fractured jaw can represent non-accidental injury. At any age, trauma, hard foods, or appliances may also cause ulceration. The lingual fraenum may be traumatised by repeated rubbing over the lower incisor teeth in cunnilingus, in recurrent coughing as in whooping cough, or in self-mutilating conditions.

Most ulcers of local cause have an obvious aetiology, are acute, usually single ulcers, last less than three weeks and heal spontaneously. Chronic trauma may produce an ulcer with a keratotic margin (Fig. 5).

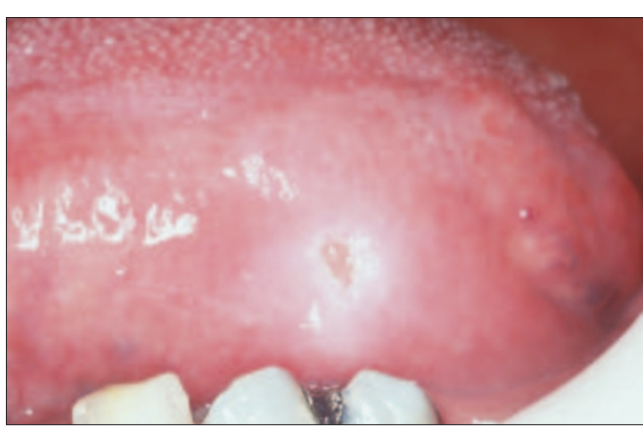

Fig. 5 Traumatic ulceration, lateral tongue

Recurrent aphthous stomatitis (RAS; aphthae; canker sores)

RAS is a very common condition which typically starts in childhood or adolescence and presents with multiple recurrent small, round or ovoid ulcers with circumscribed margins, erythematous haloes, and yellow or grey floors (Fig. 6).

RAS affects at least 20\% of the population, with the highest prevalence in higher socio-economic classes. Virtually all dentists will see patients with aphthae.

\section{Aetiopathogenesis}

Immune mechanisms appear at play in a person with a genetic predisposition to oral ulceration. A genetic predisposition is present, and there is a positive family history in about one third of patients with RAS. Immunological factors are also involved, with $\mathrm{T}$ helper cells predominating in the

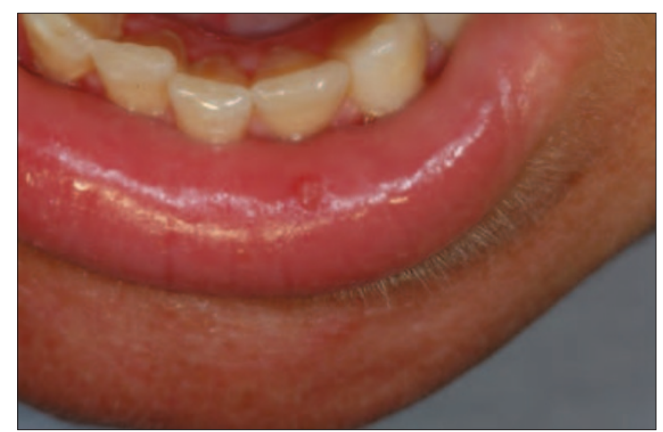

Fig. 6 Minor aphthous ulceration

RAS lesions early on, along with some natural killer (NK) cells. Cytotoxic cells then appear in the lesions and there is evidence for an antibody dependent cellular cytotoxicity (ADCC) reaction. It now seems likely therefore that a minor degree of immunological dysregulation underlies aphthae.

RAS may be a group of disorders of different pathogeneses. Cross-reacting antigens between the oral mucosa and microorganisms may be the initiators, but attempts to implicate a variety of bacteria or viruses have failed.

\section{Predisposing factors}

Most people who suffer RAS are otherwise apparently completely well. In a few, predisposing factors may be identifiable, or suspected. These include:

1. Stress: underlies RAS in many cases. RAS are typically worse at examination times.

2. Trauma: biting the mucosa, and dental appliances may lead to some aphthae.

3. Haematinic deficiency (deficiencies of iron, folic acid (folate) or vitamin $B_{12}$ ) in up to $20 \%$ of patients.

4. Sodium lauryl sulphate (SLS), a detergent in some oral healthcare products may produce oral ulceration.

5. Cessation of smoking: may precipitate or aggravate RAS.

6. Gastrointestinal disorders particularly coeliac disease (gluten-sensitive enteropathy) and Crohn's disease in about 3\% of patients.

7. Endocrine factors in some women whose RAS are clearly related to the fall in progestogen level in the luteal phase of their menstrual cycle.

8. Immune deficiency: ulcers similar to RAS may be seen in HIV and other immune defects.

9. Food allergies: underlie RAS rarely.

Drugs may produce aphthous-like lesions (see below).

Key points for dentists: aphthous ulcers

- They are so common that all dentists will see them

- It is important to rule out predisposing causes (sodium lauryl sulphate, certain foods/drinks, stopping smoking or vitamin or other deficiencies) or conditions such as Behcet's syndrome

- Enquire about eye, genital, gastrointestinal or skin lesions

- Topical corticosteroids are the main treatment 
Fig. 7 Minor aphthae

Fig. 8 Major aphthous ulceration, soft palate complex

\section{Clinical features}

There are three main clinical types of RAS, though the significance of these distinctions is unclear and it is conceivable that they may represent three different diseases:

1. Minor aphthous ulcers (MiAU; Mikulicz Ulcer) occur mainly in the 10 to 40-year-old age group, often cause minimal symptoms, and are small round or ovoid ulcers $2-4 \mathrm{~mm}$ in diameter. The ulcer floor is initially yellowish but assumes a greyish hue as healing and epithelialisation proceeds. They are surrounded by an erythematous halo and some oedema, and are found mainly on the non-keratinised mobile mucosa of the lips, cheeks, floor of the mouth, sulci or ventrum of the tongue. They are only uncommonly seen on the keratinised mucosa of the palate or dorsum of the tongue and occur in groups of only a few ulcers (one to six) at a time. They heal in seven to 10 days, and recur at intervals of one to four months leaving little or no evidence of scarring (Fig. 7).

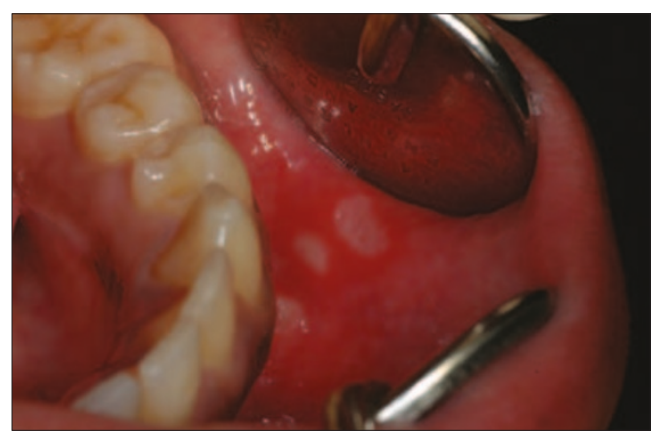

2. Major aphthous ulcers (MjAU; Sutton's Ulcers; periadenitis mucosa necrotica recurrens (PMNR)) (Figs 8 and 9) are larger, of longer duration, of more frequent recurrence, and often more painful than minor ulcers. MjAU are round or ovoid like minor ulcers, but they are larger and associated with surrounding oedema and can reach a large size, usually about $1 \mathrm{~cm}$ in diameter or even larger. They
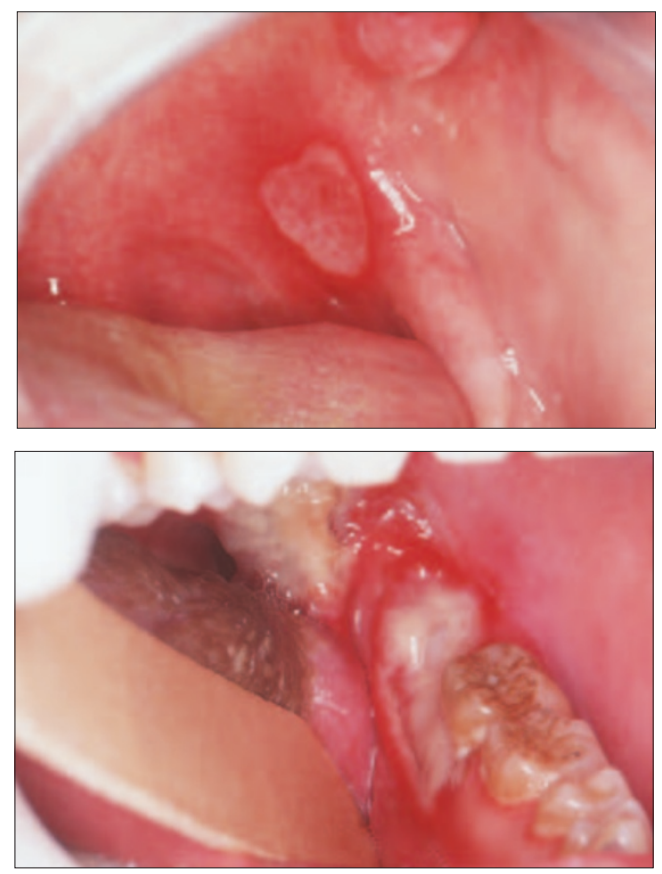

are found on any area of the oral mucosa, including the keratinised dorsum of the tongue or palate, occur in groups of only a few ulcers (one to six) at one time and heal slowly over 10 to 40 days. They recur extremely frequently may heal with scarring and are occasionally found with a raised erythrocyte sedimentation rate or plasma viscosity.

3. Herpetiform Ulceration (HU) is found in a slightly older age group than the other forms of RAS and are found mainly in females. They begin with vesiculation which passes rapidly into multiple minute pinhead-sized discrete ulcers (Fig. 10), which involve any oral site including the keratinised mucosa. They increase in size and coalesce to leave large round ragged ulcers, which heal in 10 days or longer, are often extremely painful and recur so frequently that ulceration may be virtually continuous.

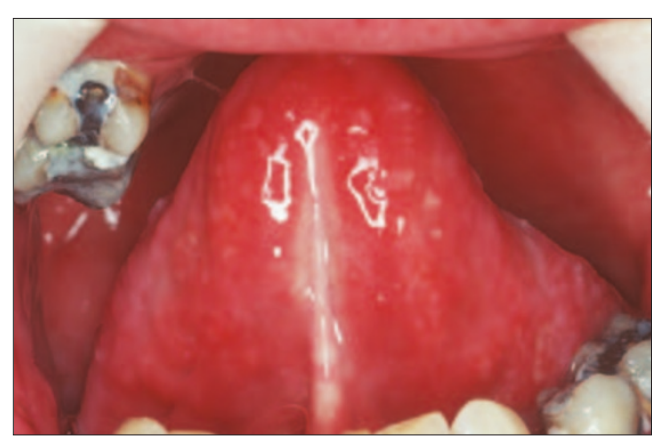

Fig. 10 Herpetiform aphthae

\section{Diagnosis}

Specific tests are unavailable, so the diagnosis must be made on history and clinical features alone. However, to exclude the systemic disorders discussed above, it is often useful to undertake the investigations shown in Table 3. Biopsy is rarely indicated, and only when a different diagnosis is suspected.

\begin{tabular}{l} 
Table 3 Investigation of aphthae \\
\hline Full blood count \\
Haematinics \\
Ferritin \\
Folate \\
$\quad$ Vitamin $B_{12}$ \\
Screen for coeliac disease
\end{tabular}

\section{Management}

Other similar disorders such as Behcet's syndrome must be ruled out (see below). Predisposing factors should then be corrected. Fortunately, the natural history of RAS is one of eventual remission in most cases. However, few patients do not have spontaneous remission for several years and although there is no curative treatment, measures should be taken to relieve symptoms, correct reversible causes (haematological disorder, trauma) and reduce ulcer duration.

\section{Maintain good oral hygiene}

Chlorhexidine or triclosan mouthwashes may help. 


\begin{tabular}{|c|c|c|}
\hline Steroid & UK trade name & $\begin{array}{l}\text { Dosage every } \\
\text { six hours }\end{array}$ \\
\hline \multicolumn{3}{|l|}{ Low potency } \\
\hline $\begin{array}{l}\text { Hydrocortisone } \\
\text { hemisuccinate pellets }\end{array}$ & Corlan & $\begin{array}{l}2.5 \mathrm{mg} \text { pellet } \\
\text { dissolved in } \\
\text { mouth close to } \\
\text { ulcers }\end{array}$ \\
\hline \multicolumn{3}{|l|}{ Medium potency } \\
\hline $\begin{array}{l}\text { Triamcinolone } \\
\text { acetonide } 0.1 \% \text { in } \\
\text { carmellose gelatin paste }\end{array}$ & $\begin{array}{l}\text { Adcortyl in } \\
\text { Orabase }\end{array}$ & $\begin{array}{l}\text { Apply paste to } \\
\text { dried lesions }\end{array}$ \\
\hline $\begin{array}{l}\text { Betamethasone } \\
\text { phosphate tablets }\end{array}$ & Betnesol & $\begin{array}{l}0.5 \text { mg; use as } \\
\text { mouthwash }\end{array}$ \\
\hline \multicolumn{3}{|l|}{ High potency } \\
\hline $\begin{array}{l}\text { Beclometasone } \\
\text { (Beclomethasone) } \\
\text { dipropionate spray }\end{array}$ & Becotide 100 & $\begin{array}{l}1 \text { puff } \\
\text { (100 micrograms) } \\
\text { to lesions }\end{array}$ \\
\hline
\end{tabular}

\section{Topical corticosteroids can usually control} symptoms

There is a spectrum of topical anti-inflammatory agents that may help in the management of RAS. Common preparations used include the following, four times daily:

- Weak potency corticosteroids topical hydrocortisone hemisuccinate pellets (Corlan), $2.5 \mathrm{mg}$ or

- Medium potency steroids - topical triamcinolone acetonide in carboxymethyl cellulose paste (Adcortyl in orabase), or betamethasone or

- Higher potency topical corticosteroids (eg beclometasone) (Table 4).

The major concern is adrenal suppression with long-term and/or repeated application, but there is evidence that $0.05 \%$ fluocinonide in adhesive paste and betamethasone-17valerate mouthrinse do not cause this problem.

Topical tetracycline (eg doxycycline), or tetracycline plus nicotinamide may provide relief and reduce ulcer duration, but should be avoided in children under 12 who might ingest the tetracycline and develop tooth staining. If RAS fails to respond to these measures, systemic immunomodulators may be required, under specialist supervision.

Key points for patients: aphthous ulcers

- These are common

- They are not thought to be infectious

- Children may inherit ulcers from parents

- The cause is not known but some follow use of toothpaste with sodium lauryl sulphate, certain foods/drinks, or stopping smoking

- Some vitamin or other deficiencies or conditions may predispose to ulcers

- Ulcers can be controlled but rarely cured

- No long-term consequences are known

Websites and patient information

http://www.usc.edu/hsc/dental/opath/Cards/Ap hthousStomatitis.html
http://openseason.com/annex/library/cic/X003 3_fever.txt.html

\section{Infections}

Infections that cause mouth ulcers are mainly viral, especially the herpesviruses, Coxsackie, ECHO and HIV viruses. Bacterial causes of mouth ulcers, apart from acute necrotising ulcerative gingivitis, are less common. Syphilis and tuberculosis are uncommon but increasing, especially in people with HIV/AIDS. Fungal and protozoal causes of ulcers are also uncommon but increasingly seen in immunocompromised persons, and travellers from the developing world.

\section{Herpes simplex virus (HSV)}

The term 'herpes' is often used loosely to refer to infections with herpes simplex virus (HSV). This is a ubiquitous virus which commonly produces lesions in the mouth and oropharynx. HSV is contracted by close contact with infected individuals from infected saliva or other body fluids after an incubation period of approximately four to seven days.

Primary infection is often subclinical between the ages of 2-4 years but may present with stomatitis (gingivostomatitis). This is usually caused by HSV-1 and is commonly attributed to 'teething' particularly if there is a fever. In teenagers or older people, this may be due to HSV-2 transmitted sexually. Generally speaking, HSV infections above the belt (oral or oropharyngeal) are caused by HSV-1 but below the belt (genital or anal) are caused by HSV-2.

The mouth or oropharynx is sore (herpetic stomatitis or gingivostomatitis): there is a single episode of oral vesicles which may be widespread, and which break down to leave oral ulcers that are initially pin-point but fuse to produce irregular painful ulcers. Gingival oedema, erythema and ulceration are prominent, the cervical lymph nodes may be enlarged and tender, and there is sometimes fever and/or malaise. Patients with immune defects are liable to severe and/or protracted infections.

HSV is neuroinvasive and neurotoxic and infects neurones of the dorsal root and autonomic ganglia. HSV remains latent thereafter in those ganglia, usually the trigeminal ganglion, but can be reactivated to result in clinical recrudescence (see below).

\section{Diagnosis}

Diagnosis is largely clinical. Viral studies are used occasionally and can include:

- culture; this takes days to give a result

- electron microscopy; this is not always available

- polymerase chain reaction (PCR) detection of HSV-DNA; this is sensitive but expensive

- immunodetection; detection of HSV antigens is of some value. 


\section{Management}

Although patients have spontaneous healing within 10-14 days, treatment is indicated particularly to reduce fever and control pain. Adequate fluid intake is important, especially in children, and antipyretics/analgesics such as paracetamol/acetoaminophen elixir help. A soft bland diet may be needed, as the mouth can be very sore. Aciclovir orally or parenterally is useful mainly in immunocompromised patients or in the otherwise apparently healthy patient if seen early in the course of the disease but does not reduce the frequency of subsequent recurrences.

\section{Recurrent HSV infections}

Up to $15 \%$ of the population have recurrent HSV-1 infections, typically on the lips (herpes labialis: cold sores) from reactivation of HSV latent in the trigeminal ganglion. The virus is shed into saliva, and there may be clinical recrudescence. Reactivating factors include fever such as caused by upper respiratory tract infection (hence herpes labialis is often termed 'cold' sores), sunlight, menstruation, trauma and immunosuppression.

Lip lesions at the mucocutaneous junction may be preceded by pain, burning, tingling or itching. Lesions begin as macules that rapidly become papular, then vesicular for about 48 hours, then pustular, and finally scab within 7296 hours and heal without scarring (Fig. 11).

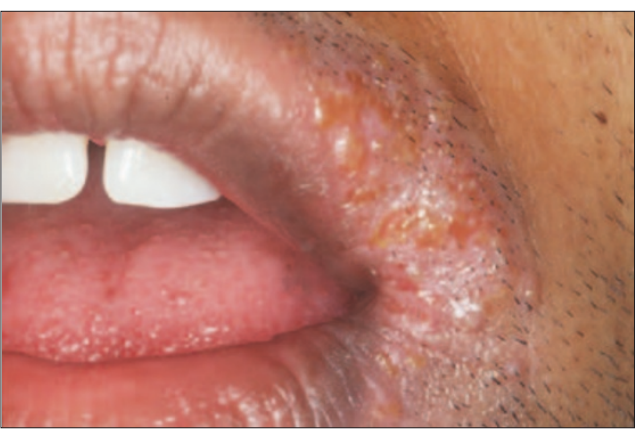

Recurrent intraoral herpes in apparently healthy patients tends to affect the hard palate or gingivae with a small crop of ulcers which heals within one to two weeks. Lesions are usually over the greater palatine foramen, following a palatal local anaesthetic injection, presumably because of the trauma.

Recurrent intraoral herpes in immunocompromised patients may appear as chronic, often dendritic, ulcers, often on the tongue.

\section{Diagnosis}

Diagnosis is largely clinical; viral studies are used occasionally.

\section{Management}

Most patients will have spontaneous remission within one week to 10 days but the condition is both uncomfortable and unsightly, and thus treatment is indicated. Antivirals will achieve maximum benefit only if given early in the disease but may be indicated in patients who have severe, widespread or persistent lesions and in immunocompromised persons. Lip lesions in healthy patients may be minimised with penciclovir 1\% cream or aciclovir 5\% cream applied in the prodrome. In immunocompromised patients, systemic aciclovir or other antivirals such as valaciclovir (the precursor of penciclovir) may be needed.

\section{Websites and patient information}

http://openseason.com/annex/library/cic/X003 3 fever.txt.html

\section{Key points for patients: cold sores}

- These are common

- They are caused by a virus (Herpes simplex) which lives in nerves forever

- They are infectious and the virus can be transmitted by kissing

- They may be precipitated by sun-exposure, stress, injury or immune problems

- They have no long-term consequences

- They may be controlled by antiviral creams or tablets, best used early on

\section{Drug-induced ulceration}

Drugs may induce ulcers by producing a local burn, or by a variety of mechanisms such as the induction of lichenoid lesions (Fig. 12). Cytotoxic drugs (eg methotrexate) commonly produce ulcers, but non-steroidal anti-inflammatory drugs (NSAIDs), alendronate (a bisphosphonate), nicorandil (a cardiac drug) and a range of other drugs may also cause ulcers.

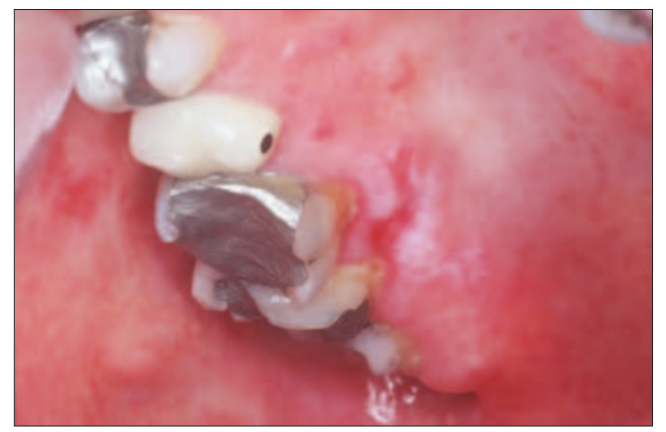

Fig. 12 Lichenoid reaction to propranolol

A drug history is important to elicit such uncommon reactions, and then the offending drug should be avoided.

Patients to refer:

- Severe aphthae

- Malignancy

- HIV-related ulceration

- TB or syphilis

- Drug-related ulceration

- Systemic disease

- Mucocutaneous disorders. 